\title{
Zone-dependent mechanical properties of human articular cartilage obtained by indentation measurements
}

\author{
J. Antons ${ }^{1}$ - M. G. M. Marascio ${ }^{2}$ J. Nohava $^{3} \cdot$ R. Martin $^{4}$ - L. A. Applegate ${ }^{5}$ - P. E. Bourban ${ }^{2}$ D. P. Pioletti $\mathbb{D}^{1}$
}

Received: 11 December 2017 / Accepted: 18 April 2018 / Published online: 4 May 2018

(c) Springer Science+Business Media, LLC, part of Springer Nature 2018

\begin{abstract}
Emerging 3D printing technology permits innovative approaches to manufacture cartilage scaffolds associated with layer-bylayer mechanical property adaptation. However, information about gradients of mechanical properties in human articular cartilage is limited. In this study, we quantified a zone-dependent change of local elastic modulus of human femoral condyle cartilage by using an instrumented indentation technique. From the cartilage superficial zone towards the calcified layer, a gradient of elastic modulus values between $0.020 \pm 0.003 \mathrm{MPa}$ and $6.44 \pm 1.02 \mathrm{MPa}$ was measured. To validate the tissue quality, the histological tissue composition was visualized by glycosaminoglycan and collagen staining. This work aims to introduce a new protocol to investigate the zone-dependent mechanical properties of graded structures, such as human articular cartilage. From this knowledge, better cartilage repair strategies could be tailored in the future.
\end{abstract}

\section{Introduction}

For functional tissue engineering, scaffolds should present appropriate mechanical strength to support loading after implantation [1] and therefore the mechanical properties of the scaffold need to match the target tissue [2]. Fabrication of such a scaffold can be complex because most tissues are not homogeneous and possess varying mechanical properties throughout their structure. With the emerging 3D printing technologies, such as biofabrication [3-5], melt electrospinning writing [6-8] and fused deposition

Electronic supplementary material The online version of this article (https://doi.org/10.1007/s10856-018-6066-0) contains supplementary material, which is available to authorized users.

$\triangle$ D. P. Pioletti

dominique.pioletti@epfl.ch

1 Laboratory of Biomechanical Orthopedics, Institute of Bioengineering, EPFL, Lausanne, Switzerland

2 Laboratory of Processing of Advanced Composites, Institute of Material Science, EPFL, Lausanne, Switzerland

3 Anton Paar, Peseux, Switzerland

4 Department of Musculoskeletal Medicine, Lausanne University Hospital (CHUV), Lausanne, Switzerland

5 Regenerative Therapy Unit, Lausanne University Hospital (CHUV), Lausanne, Switzerland moulding [9, 10], scaffolds could be produced with local mechanical properties mimicking those of tissues to be replaced.

Articular cartilage is one of these inhomogeneous tissues in which mechanical properties change steeply depending on tissue depth. The gradient of elastic modulus in cartilage is caused by the variation of tissue composition $[11,12]$. Depending on depth, four cartilage zones are distinguished [13]: (a) superficial zone (10-20\%); (b) middle zone (40-60\%); (c) deep zone (20-50\%); and (d) calcified zone (schematic found in Fig. 1). The interface between deep zone and calcified zone is the tidemark, whereas the interface between calcified zone and subchondral bone is called cement line. The zones can be described as their proportion of the overall tissue depth in percentage [14].

The zones differ on the one hand by their composition with glycosaminoglycan (GAG) and collagen (COL) content and on the other hand by collagen fiber orientation and cell density [15-17].

Although the mechanical properties of cartilage have been extensively studied, the majority of the studies focussed on the bulk properties measured from the top of the tissue (i.e., [18-20]). Only a few studies have characterized the local mechanical properties in the different zones of articular cartilage [21-23]. In other studies different techniques were used including atomic force microscopy [24] or specialized compression setups [25] to determine the zonal properties. However, the stress applied 


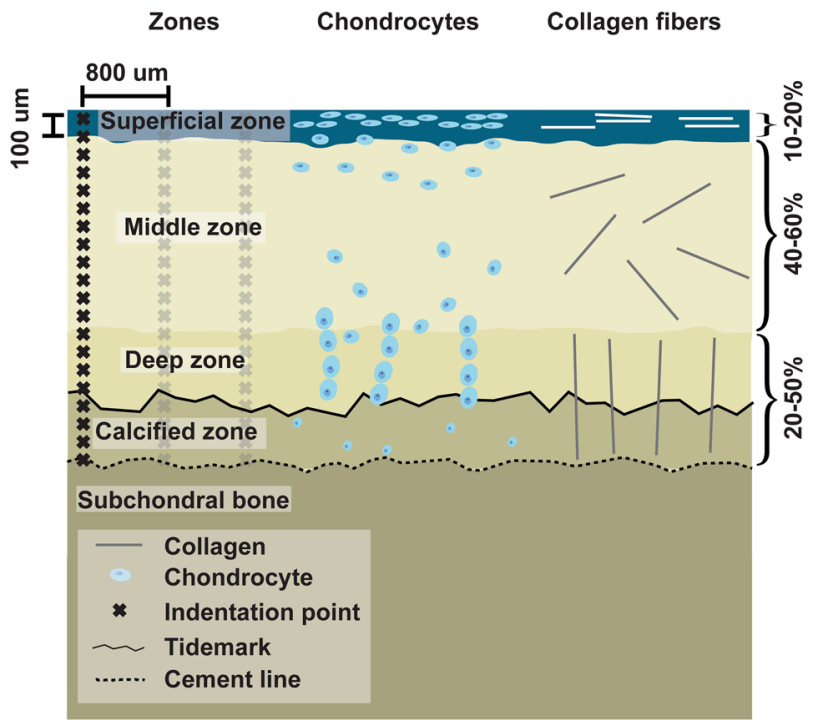

Fig. 1 Zonal structure and indentation protocol. The symbol $\mathrm{x}$ indicates the indentation point on the sample

was averaged on full thickness specimens, producing only a partial estimation of zonal cartilage mechanic response.

The scarcity of data on zonal mechanical properties in human cartilage could be explained by the technical difficulty of these kinds of measurements. To overcome this difficulty, nanoindenters exploiting a small tip to perform local indentations were adapted to measure mechanical properties of soft materials [26, 27]. Compared to atomic force microscopy, nanoindentation offers a quicker way to estimate the local mesoproperties (e.g., the local mechanical properties of the indented layer). This allows us collecting a larger ensemble of measurements in a larger area, describing more efficiently the zonal stiffness dependency.

The objective of the present study was to measure the zone-dependent elastic modulus of human cartilage tissue with a newly developed instrumented indentation technique to provide target values for future scaffold designs in tissue engineering. The meso-scale measurements (every $100 \mu \mathrm{m}$ ) are within the resolution of modern $3 \mathrm{D}$ printers, which can then adapt the scaffold properties layer-by-layer.

\section{Materials and methods}

\subsection{Human cartilage origin and preparation}

Osteochondral plugs (diameter $8 \mathrm{~mm}$ ) were harvested from the medial posterior femoral condyle of six donors (Table 1). Tissues were obtained from the DAL/CHUV biobank (Lausanne), under anonymous donation, in accordance with its regulation and approval by the Institutional Biobank. Donors 1-4 had an osteoarthritis severity grade II according to the Collins grade [28], whereas donors 5-6
Table 1 Human articular cartilage samples with donor's age, sex, and osteoarthritis grade (Collins grade)

\begin{tabular}{lllll}
\hline Donor & Age & Sex & Condition & Collins grade (I-IV) \\
\hline 1 & 62 & $\mathrm{f}$ & osteoarthritis & II \\
2 & 63 & $\mathrm{~m}$ & osteoarthritis & II \\
3 & 58 & $\mathrm{~m}$ & osteoarthritis & II \\
4 & 50 & $\mathrm{f}$ & osteoarthritis & II \\
5 & 19 & $\mathrm{~m}$ & osteochondritis dissecans & - \\
6 & 20 & $\mathrm{~m}$ & osteochondritis dissecans & - \\
\hline
\end{tabular}

were young patients having osteochondritis dissecans. The osteoarthritis stage and disease evaluations were performed by an experienced knee surgeon. Plugs were taken from visually intact regions without damage from the surgical procedure. The cartilage plugs were cut with a cutting guide using two parallel razor blades with an inter-blade distance of $3 \mathrm{~mm}$ to obtain flat cartilage surfaces. The remaining tissue was used for histological analysis. For the instrumented indentation measurements, the sample was glued onto a $35 \mathrm{~mm}$ petri dish with the cross-section facing the indenter using a small drop of instant adhesive (Loctite 401, Henkel, Germany). Samples were frozen at $-20^{\circ} \mathrm{C}$ and stored until further use. Before the measurement, the sample was thawed for $2 \mathrm{~h}$ in phosphate buffered saline solution at $22{ }^{\circ} \mathrm{C}$. The sample was completely immersed in saline solution supplemented with $1 \%$ pentamycin/streptomycin to preserve hydration of the tissue and to avoid degradation during the measurements. Under these conditions it was previously demonstrated that the mechanical properties of articular cartilage are not influenced by one freeze/ thaw cycle [29].

\subsection{Indentation measurements}

The indentation tests were performed by a specifically developed indenter (Bioindenter ${ }^{\mathrm{TM}}$, Anton Paar, Peseux, Switzerland). This indenter was adapted to measure the mechanical properties of soft materials. All indentation tests were implemented in displacement control mode using a spherical indenter tip (diameter $500 \mu \mathrm{m}$ ) made of ruby. The maximum depth of $20 \mu \mathrm{m}$ was attained at a displacement rate of $120 \mu \mathrm{m} / \mathrm{min}$. The loading was followed by a $30 \mathrm{~s}$ hold-time and $120 \mu \mathrm{m} / \mathrm{min}$ unloading rate leading to a relaxation response (Fig. 2).

The final objective of such measurements is to reproduce the progressive stiffening by additive manufacturing (AM). In particular, Fused Deposition Modelling / Fused Filament Fabrication (FDM/FFF) was selected as method of choice among the AM technologies. By FDM/FFF, features up to $50-200 \mu \mathrm{m}$ can be reproduced in $z$-direction [30]. Therefore, an indentation pace of $100 \mathrm{um}$ was defined to match the manufacturing limits. The first indentation was 


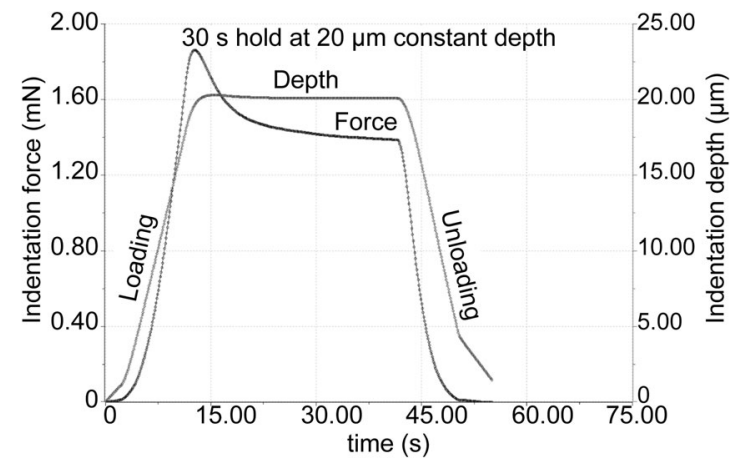

Fig. 2 Relaxation response of human femoral condyle cartilage. Example of indentation at $120 \mu \mathrm{m} / \mathrm{s}$ loading rate, $30 \mathrm{~s}$ hold and 120 $\mu \mathrm{m} / \mathrm{s}$ unloading rate

performed $100 \mu \mathrm{m}$ from the superficial zone towards the bone as indicated in Fig. 1. Subsequently, a line consisting of 11-40 indentations, spaced by $100 \mu \mathrm{m}$ was performed on cross-sectioned cartilage towards the bone. Three parallel indentation lines with a gap of $800 \mu \mathrm{m}$ were measured. A typical indentation measurement on articular cartilage is illustrated in Fig. 3. The relaxation behaviour of articular cartilage during the $30 \mathrm{~s}$ hold-time is shown in Fig. 2.

The elastic modulus was calculated according to Hertz's model for contact of a sphere with a flat surface by fitting the loading curve (Fig. 4) by Eq. 1 (modified from [31]):

$F=\frac{4}{3} \cdot E_{r} \sqrt{R} \cdot\left(h-h_{\text {offset }}\right)^{\frac{3}{2}}$

In Eq. $1 h$ is the indentation depth, $R$ is the radius of the indenter, $E_{r}$ is the reduced elastic modulus and $F$ is the maximum load during the indentation. The $h_{\text {offset }}$ accounts for the initial establishment of "soft" contact that resulted from small local irregularities (unevenness) of the surface. An example of this initial phase of contact establishment is shown in Fig. 4. Since this initial phase of contact is not representing the properties of the cartilage tissue, it was decided to fit the loading portion of the indentation curve after the correction $h_{\text {offset }} . h_{\text {offset }}$ is the distance required by the indenter to establish reliable "soft" contact and can be indicated by rapid increase in the force-distance curve.

Linearized fit of the loading portion of the indentation curve was done using Eq. 1 with proprietary Indentation software (Anton Paar, Indentation V7.3.9, Peseux, Switzerland), where $h_{\text {offset }}$ is representing the initial phase of 'soft' contact (i.e., not representing the tested tissue).

\subsection{Relative depth}

The relative depth of the cartilage was determined by normalizing the cartilage thickness of the sample to the indentation point. The start of the cartilage superficial layer

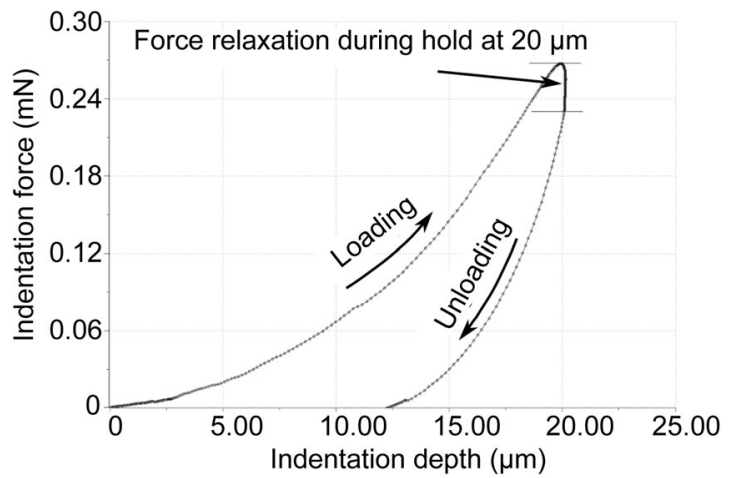

Fig. 3 Example of indentation measurement. Indentation was performed on donor 4, Indentation 17

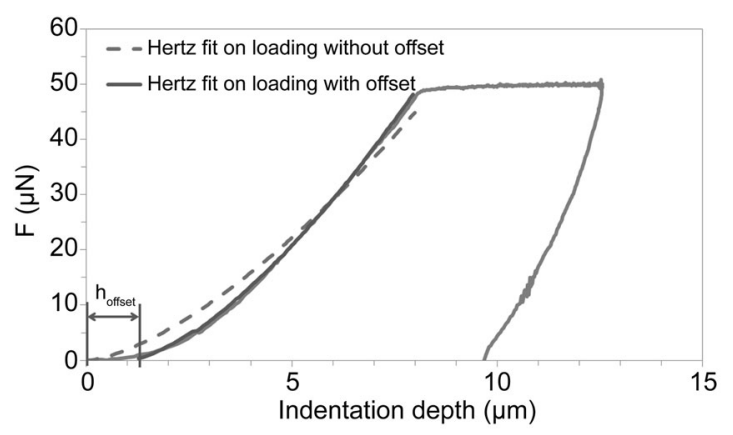

Fig. 4 Two indentation curves of the initial phase of contact without and with offset to correct for tissue surface irregularities

is $0 \%$ and the start of the tidemark is $100 \%$. The cartilage thickness was measured from the histological section at five randomly chosen points from the cartilage surface to the tidemark with Image $\mathbf{J}$ (version $1.48 \mathrm{v}$ ) and subsequently averaged. Zones are defined according to their relative proportion as stated in the literature [14]. The superficial zone has a relative proportion between 10-20\%, the middle zone with $40-60 \%$ and the deep zone at $20-50 \%$. The precise localization of the cartilage zones is therefore challenging and the indication in Fig. 5 serves as an estimate.

\subsection{Histology}

The samples were fixed in $4 \%$ paraformaldehyde at $22{ }^{\circ} \mathrm{C}$ overnight. Thereafter, the bone was decalcified by a solution consisting of $20 \%$ Tri-sodium citrate in acetic acid for $48 \mathrm{~h}$ at $22^{\circ} \mathrm{C}$. This was followed by dehydration in ethanol and embedding into paraffin following standard histological protocols. Sections with a thickness of $5 \mu \mathrm{m}$ were cut with a microtome and stained for standard Hematoxylin and Eosin, for sulphated GAGs with Alcian Blue staining (SigmaAldrich, Buchs, Switzerland) and for COL with Picrosirius Red staining (Sigma-Aldrich, Buchs, Switzerland). The 


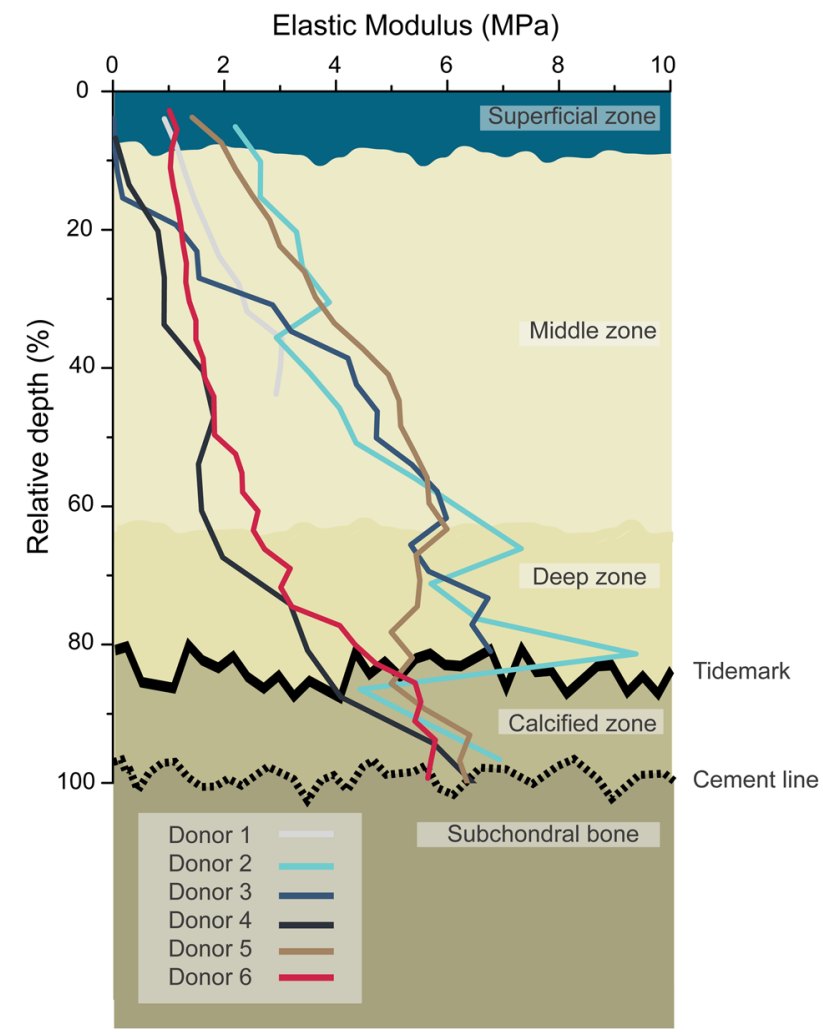

Fig. 5 Depth-dependent elastic modulus of human femoral condyle cartilage. The relative depth corresponds to the distance between the superficial layer $(0 \%)$ and subchondral bone $(100 \%)$ and was normalized for each sample. Donor 1 and 3 were not measured to $100 \%$ since the fixed number of indentations did not reach to the bone. Donor 2 was not considered for elastic modulus values, but kept to show gradient; $n=3$; standard deviation was omitted to visualize trend (displayed in supplementary S1)

histological sections were analysed with a Leica DM5500 (Wetzlar, Germany) microscope. A tile scan for measurements and images was performed with the $5 \times$ magnification including an automatic stitching of the images. The close up image was taken at $20 \times$ magnification.

\subsection{Quantification of histology sections}

The cell count was determined by acquiring images in 10 randomly selected regions of each sample in $20 \times$ magnification $(539.35 \times 404.51 \mu \mathrm{m})$, counting the cells manually supported by ImageJ (version $1.48 \mathrm{v}$ ) and its plugin CellCounter.

\section{Results}

\subsection{Indentation measurements}

In all cartilage samples, the indentation measurements highlighted a gradient of elastic modulus with an increase of values from $0.020 \pm 0.003 \mathrm{MPa}$ at the cartilage superficial zone to $6.44 \pm 1.02 \mathrm{MPa}$ at the calcified layer (Fig. 5). The gradient intensity deviated between the six donors, but showed the same common tendency.

All samples were normalized to their relative depth (distance between cartilage superficial layer and tidemark) to account for different thicknesses. Donor one and three were not measured up to the tidemark due to a fixed number of indentations in the indentation protocol.

\subsection{Histological evaluation}

The cell distribution and cell shape in the human cartilage tissue visually corresponded to healthy cartilage and thus confirmed the quality within the harvesting site (Fig. 6a). The intensity of Alcian Blue (AB) staining and thus the GAG content increased with depth from the cartilage superficial layer towards the tidemark (Fig. 6b). A low intensity ( $\mathrm{L}$ ) $\mathrm{AB}$ staining was observed at the cartilage surface, whereas towards the calcified layer the staining intensity increased $(\mathrm{H})$. The GAG content was in general lower in the superficial zone, increased with depth in the middle layer and decreased in the deep layer towards the tidemark. High GAG content was additionally visible in close contact to the chondrocytes themselves (Fig. 6f), interpreted as continuous GAG synthesis of intact chondrocytes in the osteoarthritis affected cartilage. In Picrosirius Red staining (SR), the staining for collagen, the trend was inversed indicating a high $(\mathrm{H})$ intensity staining at the surface and lower intensity (L) towards the calcified layer. For SR the staining intensity was high at the surface and decreased towards the calcified layer (Fig. 6c). Concerning the collagen content, the superficial layer showed a mild fibrillation and degeneration of collagen fibres (Fig. 6g), but a stable portion of the cartilage matrix in the middle layer and a decrease in the deep layer towards the tide mark. The collagen fiber orientation was visualized under polarized light. The parallel orientation of the collagen fibers in the superficial layer was evidenced by the different color under polarized light (Figs. 6d, h). Moreover, the thickness of the articular cartilage layer and cell count was measured (Table 2). The average cell count was very similar for all samples ranging from $32.70 \pm 7.53$ to $47.2 \pm$ 10.51 cells. The thickness of the articular cartilage ranged from $1484.00 \pm 75.23 \mu \mathrm{m}$ to $3624.40 \pm 164.11 \mu \mathrm{m}$ between patients.

\section{Discussion}

Only a few studies have characterized the mechanical properties in the different zones of articular cartilage and most of these studies focus on animal samples [24, 25]. In 


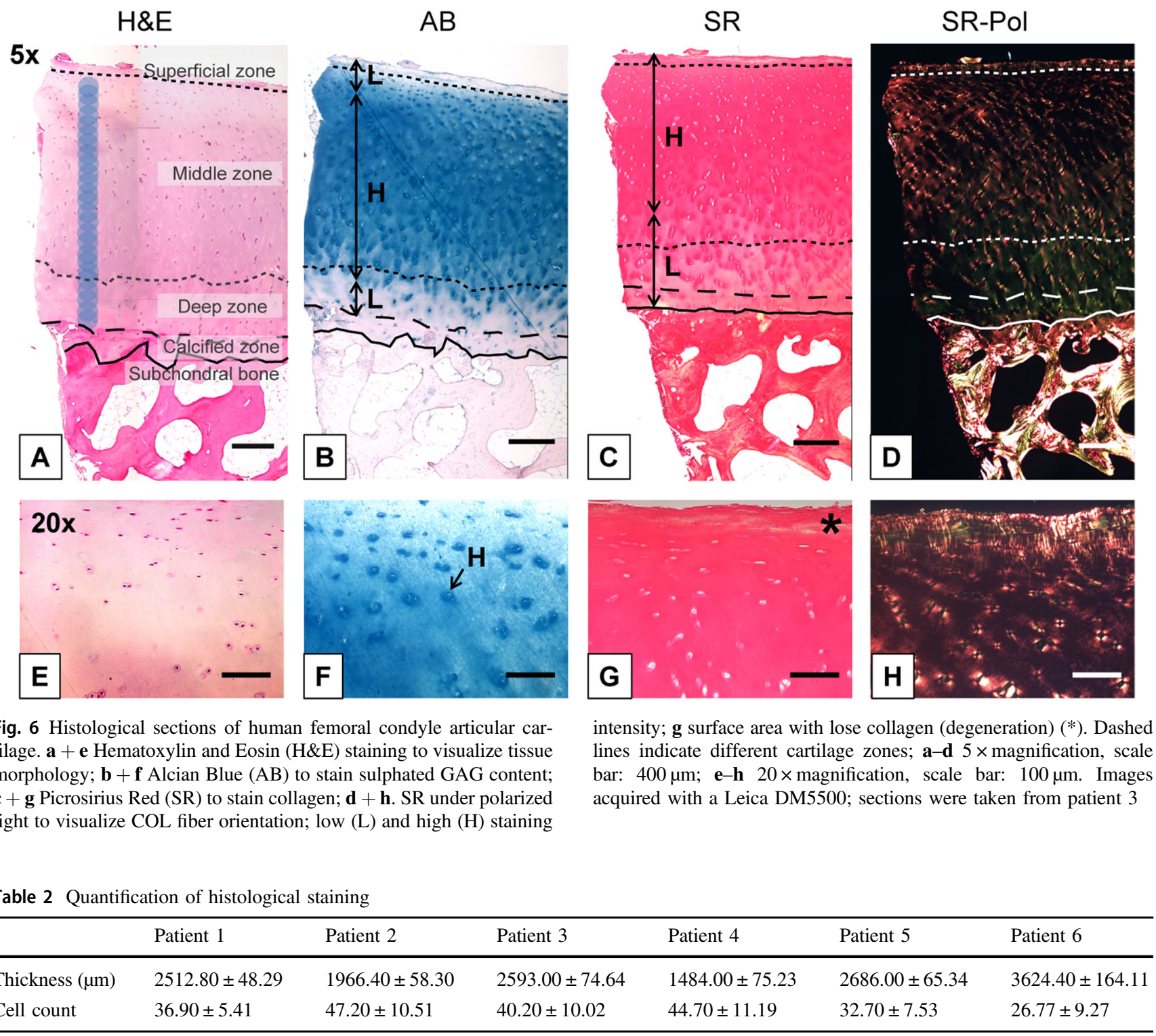

addition, the limitations in previous studies are pretreatments of the samples by paraformaldehyde (fixation), ethanol (dehydration) or embedding (PMMA) before the measurement $[32,33]$. These pre-treatments change the mechanical properties of the samples. The novelty of the present work is the zonal measurement of six human articular cartilage samples by instrumented indentation in wet condition without any pre-treatment. The measured local elastic modulus was then compared to the local tissue composition obtained by histological staining.

We successfully measured zonal elastic modulus of human femoral condyle cartilage using nanoindentation and found that elastic modulus increased with depth. This work compares well to previous work of other studies where the zonal properties of human articular cartilage were inferred from bulk compression measurements [34]. Indeed, in this mentioned study, an increasing gradient of elastic modulus with increasing depth was also found. Moreover, although their measurements were performed on the cartilage of the femoral head, the range of elastic modulus was comparable to our results. They reported an equilibrium confined compression modulus between $1.16 \pm 0.20 \mathrm{MPa}$ in the cartilage superficial layer to $7.75 \pm 1.45 \mathrm{MPa}$ in the deep layer while we measured between $0.020 \pm 0.003 \mathrm{MPa}$ and $6.44 \pm$ $1.02 \mathrm{MPa}$ from cartilage superficial zone towards the calcified layer by nanoindentation. Even though our measurements were performed on the cartilage cross-section we hypothesize that the elastic modulus should be very similar to measurements from the top. Our hypothesis is based on a previous study which discovered that measuring local mechanical properties by atomic force microscopy from the cross-section or the top of porcine articular cartilage 
samples made a difference of up to $20 \%$ in elastic modulus (superficial: 20\% lower, middle: $14 \%$ lower, deep: no difference) [35]. In contrast to our study, they observed a decreasing elastic modulus towards the deeper zones of articular cartilage. This could be due to the different length scale in which they measured since indentations were performed with a diameter of $5 \mu \mathrm{m}$ tip in small regions of $20 \times$ $20 \mu \mathrm{m}$, whereas our measurements were in the meso-scale performed with $500 \mu \mathrm{m}$ diameter tip and $100 \mu \mathrm{m}$ distance between indentations. Another reason for the gradient difference could be that they measured on porcine instead of human articular cartilage.

To validate the quality of the human cartilage tissue, we assessed the zone-dependent GAG and COL content by histochemical analysis (Fig. 6) (Alcian Blue and Picrosirius Red). The GAG and COL content of the samples changed according to depth. The collagen portion of the matrix remained stable in all samples while the GAG content varied from low in the superficial layer to high in the middle layer and lowered again towards the calcified layer.

The collagen fibers are responsible for the tensile properties of cartilage [15] and have shown to only have little effect on the compressive properties. Nevertheless, it has been demonstrated that collagen fibers could indicate tissue quality and onset of osteoarthritis [36]. Since we have noticed a mild fibrillation of collagen in the superficial layer, this might indicate a degeneration in the samples, but mostly in the superficial layer.

We observed that GAG content increased from the superficial zone towards the middle zone and diminished between middle and deep zone, whereas at the same time elastic modulus increased from the superficial zone towards the subchondral bone. The increase in elastic modulus between middle and deep zone was thus not solely caused by the GAG content. We assume that the elastic modulus increase in the deep zone might have been due to a higher collagen content and collagen fiber diameter [16]. The same assumption for the deep layer of articular cartilage has already been made in another study in which zonal mechanical properties were measured [34].

In general, special care should be taken when measuring materials with significant modulus heterogeneity with indentation (such as at the cartilage-bone interface). It has previously been demonstrated that in the case of a material heterogeneity, the indenter size should be less than $10 \%$ of the expected length scale of modulus changes in order to obtain reliable results [37]. The measurements in the present work were focussed on the articular cartilage zones and not on the subchondral bone. Thus, the criterion of having an indenter size of less than $10 \%$ of the expected length scale of modulus does not necessarily have to be followed for our measurements since a difference in the order of 2 in magnitude of elastic modulus is not observed.
Even though the mechanical gradient was comparable to existing measurements, some aspects and limitations of the technique used here should be considered. Due to its nature of testing relatively small areas (around $200 \mu \mathrm{m}$ diameter) instrumented indentation is quite sensitive to local differences and hence to sample degradation, sample preparation, and the surrounding environment. An approximation of the area involved in the indentation response can be done via contact radius. For elastic spherical indentation the contact radius $a$ is $\sim \sqrt{ } \mathrm{Rh}$ where $R$ is the radius of the indenter and $h$ is the indentation depth. For this study this corresponds to a contact radius of $\sim 100 \mu \mathrm{m}$. We chose a contact area of 100 $\mu \mathrm{m}$ and a space between indentation points of $100 \mu \mathrm{m}$ to get a full coverage of all zones and a maximum of information about the zonal properties. We assume that the cartilage deformation is only elastic (i.e., it fully recovers after unloading) as we found very similar results when performing two consecutive measurements on the same spot.

The measurement of the superficial zone is particularly challenging since the indentation is near the edge and edgeeffects could introduce artefacts into the measurements [38, 39]. This can be partially overcome by indentation from the top surface. However, indenting from the top surface would only help measuring the local bulk properties of the tissue (e.g., the average properties of all the layers). Separate measurements of previously dissected layers should be difficult, because according to the Mow model [40], the measured cartilage properties are dependent on the cartilage thickness and the underlying substrate. The local character of the instrumented indentation method used in this work might explain the standard deviation for the measurements in supplemental data (S1). For each patient three indentation lines were performed and averaged. These parallel lines were spaced by $800 \mu \mathrm{m}$. It is possible that the chosen distance between the indentation lines was too large and the elastic modulus in the adjacent lines already changed due to different structure or composition. For a better comparison between samples we normalized the depth to the distance between cartilage surface layer and calcified layer. Unfortunately, not all samples were measured up to the calcified layer. This was due to the fixed indentation procedure which did not account for different thicknesses of the cartilage. However, the same common trend (i.e., increase of elastic modulus towards the bone) can be observed in all samples. The sample degradation, the age of the donor, the disease state or the site in which the sample was harvested could also influence the measurement. Although we took care to include only visually intact cartilage in this study, it is virtually impossible to exclude degradation of the microstructure especially on the surface. It was also difficult to harvest the samples from precisely the same region of the femoral condyle. This was caused by the degeneration state of the tissue and the surgical procedure which left regions 
damaged by cuts and burns. In addition to the aforementioned issues, it was technically difficult to measure the sample in precise depths from the top surface, which led us to measure the sample from the cross-section.

Instrumented indentation would enable engineers to obtain local mechanical data about their target tissue to better match the properties of their scaffold design to the target tissue. The local elastic modulus of human femoral condyle articular cartilage measured in this work could thus be useful to provide target values for future scaffold designs. The values can be registered in a 3D printer which adapts the scaffold properties layer-by-layer. This approach would not only be limited to articular cartilage, but has the potential to improve the scaffold design for several organs that possess defined local mechanical properties.

Acknowledgements The authors would like to thank the Swiss National Science Foundation (\#200021_143413) for their financial support. Furthermore, the authors would like to thank Dr. Nadine Stokar for her help with histology interpretation, and Mme Sandra Jaccoud and Dr. Virginie Philippe for assistance with the DAL Biobank.

\section{Compliance with ethical standards}

Conflict of Interest The authors declare that they have no conflict of interest

\section{References}

1. Guilak F, Butler DL \& Goldstein SA. Functional tissue engineering: the role of biomechanics in articular cartilage repair. Clin Orthopaed Rel Res. 2001;391 Suppl: S295-305.

2. Hutmacher DW. Scaffolds in tissue engineering bone and cartilage. Biomaterials. 2000;21:2529-43.

3. Mouser VHM, et al. Three-dimensional bioprinting and its potential in the field of articular cartilage regeneration. Cartilage. 2016. p. 1947603516665445.

4. Visser $J$, et al. Biofabrication of multi-material anatomically shaped tissue constructs. Biofabrication. 2013;5:35007.

5. Zadpoor AA, Malda J. Additive manufacturing of biomaterials, tissues, and organs. Annal Biomed Eng. 2016;45:1-11.

6. Dalton PD, et al. Patterned melt electrospun substrates for tissue engineering. Biomed Mater. 2008;3:34109.

7. Brown TD, Dalton PD, Hutmacher DW. Direct writing by way of melt electrospinning. Adv Mater. 2011;23:5651-7.

8. Brown TD, Dalton PD, Hutmacher DW. Melt electrospinning today: an opportune time for an emerging polymer process. Prog Polym Sci. 2015;56:116-166.

9. Zein I, et al. Fused deposition modeling of novel scaffold architectures for tissue engineering applications. Biomaterials. 2002;23:1169-85.

10. Chia HN, Wu BM. Recent advances in 3D printing of biomaterials. J Biol Eng. 2015;9:4.

11. Jeon J, Malda J, Schrobback K, Hutmacher DW, Klein T. Methods in Bioengineering: 3D Tissue Engineering. In: Berthiaume F, Morgan JR, editors. Artech House; 2010. p. 205-224..

12. Mow VC, Zhu W, Ratcliffe A. Structure and function of articular cartilage and meniscus. Basic orthopaedic biomechanics. New York, NY: Raven Press; 1991. p. 143-1998.
13. Temenoff JS, Mikos AG. Review: tissue engineering for regeneration of articular cartilage. Biomaterials. 2000;21:431-40.

14. Athanasiou KA, Darling EM, Hu JC. Articular cartilage tissue engineering. Synthesis Lectures on Tissue Engineering 1.1 2009:1-182.

15. Kempson GE, Freeman MAR, Swanson SAV, Tensile. Nature. 1968;220:1127-8.

16. Muir $\mathrm{H}$, Bullough $\mathrm{P}$, Maroudas A. The distribution of collagen in human articular cartilage with some of its physiological implications. J Bone Joint Surg. 1970;52:554-63.

17. Stockwell RA. The cell density of human articular and costal cartilage. J Anat. 1967;101:753-63.

18. Laasanen MS, et al. Biomechanical properties of knee articular cartilage. Biorheology. 2003;40:133-40.

19. Mow VC, Holmes MH, Michael Lai W. Fluid transport and mechanical properties of articular cartilage: a review. J Biomech. 1984;17:377-94.

20. Armstrong CG, Mow VC. Variations in the intrinsic mechanical properties of human articular cartilage with age, degeneration, and water content. J Bone Joint Surg. 1982;64:88-94.

21. Ebenstein DM, et al. A nanoindentation technique for functional evaluation of cartilage repair tissue. J Mater Res. 2011;19:273-81.

22. Franke $\mathrm{O}$, et al. Dynamic nanoindentation of articular porcine cartilage. Mater Sci Eng C. 2011;31:789-95.

23. Franke $\mathrm{O}$, et al. Mechanical properties of hyaline and repair cartilage studied by nanoindentation. Acta Biomater. 2007;3:873-81.

24. Tomkoria S, Patel RV, Mao JJ. Heterogeneous nanomechanical properties of superficial and zonal regions of articular cartilage of the rabbit proximal radius condyle by atomic force microscopy. Med Eng Phys. 2004;26:815-22.

25. Chen AC. et al. Depth-and strain-dependent mechanical and electromechanical properties of full-thickness bovine articular cartilage in confined compression. J Biomech. 2001;34:1-12.

26. Eberwein $P$, et al. Nanoindentation derived mechanical properties of the corneoscleral rim of the human eye. Key Eng Mater. 2014;606:117-120.

27. Swain MV, Nohava J, Eberwein P. A simple basis for determination of the modulus and hydraulic conductivity of human ocular surface using nano-indentation. Acta Biomater. 2016;50:312-321.

28. Collins D. The pathology of articular and spinal diseases. London: Edward Arnold; 1949. p. 76-115.

29. Szarko M, Muldrew K, Bertram JE. Freeze-thaw treatment effects on the dynamic mechanical properties of articular cartilage. BMC Musculoskelet Disord. 2010;11:231.

30. Wang $X$, et al. 3D printing of polymer matrix composites: a review and prospective. Compos Part B Eng. 2017;110:442-458.

31. Last $J$, et al. Determining the mechanical properties of human corneal basement membranes with atomic force microscopy. J Struct Biol. 2009;167:19-24.

32. Gupta HS, et al. Two different correlations between nanoindentation modulus and mineral content in the bone-cartilage interface. J Struct Biol. 2005;149:138-148.

33. Campbell SE, Ferguson VL, Hurley DC. Nanomechanical mapping of the osteochondral interface with contact resonance force microscopy and nanoindentation. Acta Biomater. 2012;8: 4389-4396.

34. Chen SS, Falcovitz YH, Schneiderman R, Maroudas A, Sah RL. Depth-dependent compressive properties of normal aged human femoral head articular cartilage: relationship to fixed charge density. Osteoarthritis and Cartilage 9.6 2001;561-9.

35. McLeod Ma, Wilusz RE, Guilak F. Depth-dependent anisotropy of the micromechanical properties of the extracellular and pericellular matrices of articular cartilage evaluated via atomic force microscopy. J Biomech. 2013;46:586-592. 
36. Hollander AP, et al. Damage to type II collagen in aging and osteoarthritis starts at the articular surface, originates around chondrocytes, and extends into the cartilage with progressive degeneration. J Clin Investig. 1995;96:2859-2869.

37. Armitage OE, Oyen ML. Indentation across interfaces between stiff and compliant tissues. Acta Biomater. 2017;56:36-43.

38. Jakes JE, et al. Nanoindentation near the edge. J Mater Res. 2009;24:1016-1031.
39. Jakes JE, Stone DS. The edge effect in nanoindentation. Philos Mag. 2011;91:7-9.

40. Mow VC, et al. Biphasic indentation of articular cartilage-II. A numerical algorithm and an experimental study. J Biomech. 1989;22:853-861. 